\title{
Frequency-Dependent Squeezing for Advanced LIGO
}

\author{
L. McCuller $\odot,{ }^{1, *}$ C. Whittle $\odot,{ }^{1, \dagger}$ D. Ganapathy $\odot,{ }^{1}$ K. Komori $\odot,{ }^{1}$ M. Tse $\odot,{ }^{1}$ A. Fernandez-Galiana $\odot,{ }^{1}$ L. Barsotti $\odot,{ }^{1}$ \\ P. Fritschel@ ${ }_{1}^{1}$ M. MacInnis, ${ }^{1}$ F. Matichard $\odot,{ }^{1,2}$ K. Mason, ${ }^{1}$ N. Mavalvala, ${ }^{1}$ R. Mittleman, ${ }^{1}$ Haocun Yu $\odot,{ }^{1}$ \\ M. E. Zucker, ${ }^{1,2}$ and M. Evans ${ }^{1}$ \\ ${ }^{1}$ LIGO, Massachusetts Institute of Technology, Cambridge, Massachusetts 02139, USA \\ ${ }^{2}$ LIGO, California Institute of Technology, Pasadena, California 91125, USA
}

(Received 23 February 2020; accepted 23 March 2020; published 28 April 2020)

\begin{abstract}
The first detection of gravitational waves by the Laser Interferometer Gravitational-Wave Observatory (LIGO) in 2015 launched the era of gravitational-wave astronomy. The quest for gravitational-wave signals from objects that are fainter or farther away impels technological advances to realize ever more sensitive detectors. Since 2019, one advanced technique, the injection of squeezed states of light, is being used to improve the shot-noise limit to the sensitivity of the Advanced LIGO detectors, at frequencies above $\sim 50 \mathrm{~Hz}$. Below this frequency, quantum backaction, in the form of radiation pressure induced motion of the mirrors, degrades the sensitivity. To simultaneously reduce shot noise at high frequencies and quantum radiation pressure noise at low frequencies requires a quantum noise filter cavity with low optical losses to rotate the squeezed quadrature as a function of frequency. We report on the observation of frequencydependent squeezed quadrature rotation with rotation frequency of $30 \mathrm{~Hz}$, using a 16-m-long filter cavity. A novel control scheme is developed for this frequency-dependent squeezed vacuum source, and the results presented here demonstrate that a low-loss filter cavity can achieve the squeezed quadrature rotation necessary for the next planned upgrade to Advanced LIGO, known as "A+."
\end{abstract}

DOI: $10.1103 /$ PhysRevLett.124.171102

Introduction.-Quantum noise imposes a fundamental limitation on the precision of physical measurements. In gravitational-wave detectors such as Advanced LIGO [1], it manifests in two ways: shot noise, caused by quantum fluctuations in the arrival time of photons detected at the interferometer output, and quantum radiation pressure noise, due to quantum fluctuations in the photon flux impinging on the interferometer mirrors. Both noises have a common origin-electromagnetic vacuum states entering the open output port of the interferometer [2].

During its third observing run, Advanced LIGO has been detecting roughly one astrophysical event per week, compared to one per month in previous observing runs. A significant factor in the sensitivity improvements that has enabled this increased detection rate is the injection of squeezed states of light into the output port of the Advanced LIGO detectors [3]. Squeezed state injection-or simply "squeezing"- has also been used in the Advanced Virgo and GEO detectors $[4,5]$. The squeezed states used redistribute quantum uncertainty to have a minimum in the phase quadrature, thus reducing shot noise that dominates above

Published by the American Physical Society under the terms of the Creative Commons Attribution 4.0 International license. Further distribution of this work must maintain attribution to the author(s) and the published article's title, journal citation, and DOI. $\sim 50 \mathrm{~Hz}$. As a consequence of the Heisenberg uncertainty principle, the increased noise in the amplitude quadrature degrades the sensitivity of the interferometer at lower frequencies due to radiation pressure that drives motion of the $40 \mathrm{~kg}$ mirrors of the interferometer. This quantum radiation pressure noise has been observed in the Advanced LIGO [6] and Virgo [7] detectors under current operating conditions, and limits the level of squeezing which can be profitably used.

To avoid degradation in the low frequency sensitivity due to the quantum radiation pressure noise that results from higher levels of squeezing and/or increased laser power in the interferometers, a technique known as "frequencydependent squeezing" [8,9] is required [10,11]. In this work, we present a measurement of frequency-dependent squeezing from a system scalable to Advanced LIGO's upcoming $\mathrm{A}+$ upgrade. To achieve this, an optical cavity with low optical losses realized a squeezing rotation frequency nearly 2 orders of magnitude lower than the previous demonstration [12]. We also introduce a novel optical sensing scheme that allows for controlling the squeezed vacuum source to meet the stringent requirements of the Advanced LIGO detectors [13]. The importance of frequency-dependent squeezing for gravitational-wave detection cannot be overstated. $\mathrm{A}+$ is the only planned upgrade for Advanced LIGO in the foreseeable future, and frequency-dependent squeezing the only technology mature enough to provide broadband improvement in sensitivity. 


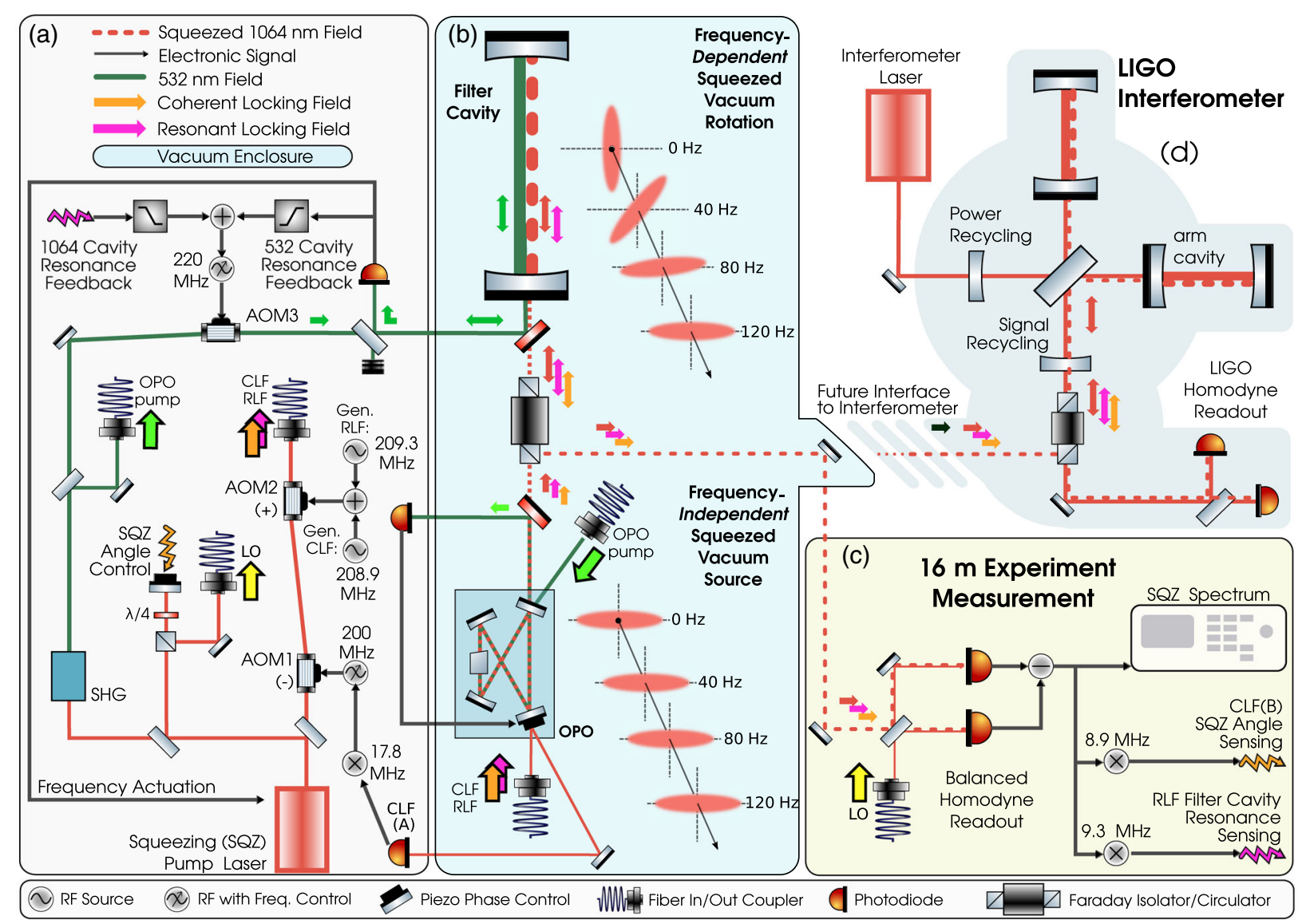

FIG. 1. Schematic overview of the optical and electronic layout for the experiment. (a) The output of a $1064 \mathrm{~nm}$ laser is used to produce a $532 \mathrm{~nm}$ field via a second harmonic generator (SHG), a local oscillator (LO) for homodyne detection, and two frequencyshifted fields (CLF and RLF) for generating error signals to control the squeeze angle and the filter cavity detuning. The squeezing angle is sensed using the coherent locking field (CLF), and the filter cavity detuning is sensed by the resonant locking field (RLF). The $532 \mathrm{~nm}$ field is split into two components, one for generating squeezing as well as controlling the length of the OPO, and the other for controlling the length of the filter cavity. (b) Frequency-independent squeezed vacuum generated by the OPO is injected into the filter cavity, and experiences frequency-dependent rotation upon reflection. (c) The frequency-dependent squeezing is measured on a balanced homodyne detector. (d) How the filter cavity will integrate optically with the LIGO interferometer.

Concurrent with, and independent of, this work, a similar experiment was carried out in Japan with a $300 \mathrm{~m}$ filter cavity, which demonstrates sub-shot-noise performance below the squeezed state rotation frequency [14].

Frequency-dependent squeezing with a filter cavity.We produce frequency-dependent squeezing by reflecting a squeezed state from one or more optical cavities, known as filter cavities, a method first suggested by Kimble et al. in 2001 [8]. When the filter cavity resonance is detuned with an offset relative to the carrier frequency of the squeezed state, it applies a differential phase shift to the upper and lower sidebands of the squeezed optical field. Since the squeezed quadrature angle at a given sideband frequency is determined by the relative phase between the upper and lower sidebands, the filter cavity serves to rotate the squeezed state for sideband frequencies that lie within the cavity linewidth (see Appendix A.3 of Ref. [9] for details).
For Advanced LIGO, a single filter cavity is needed to imprint the required frequency dependence on the injected squeezing $[10,15]$. When squeezing is injected into the interferometer without a filter cavity, as is routinely done in both Advanced LIGO [3] and Advanced Virgo [4] during observing run 3 , the optomechanical interaction between the amplitude of the optical field and the momentum of the suspended optics will shear (both squeeze and rotate) the injected state at low frequencies. When injecting frequencyindependent squeezing, this rotates part of the antisqueezed vacuum noise into the readout quadrature [16]. The A+ filter cavity is designed to apply a compensating rotation to the squeezed state before it enters the interferometer, to cancel the rotation due to the optomechanical interaction.

The power circulating in the interferometer arm cavities determines the strength of the optomechanical interaction and thus the frequency of the squeeze angle rotation 
required to compensate it. With up to $800 \mathrm{~kW}$ of circulating power anticipated for future observation runs, the filter cavity must have a bandwidth of approximately $50 \mathrm{~Hz}$, equivalent to a storage time of $3 \mathrm{~ms}$.

In general, for a low-loss optical resonator of length $L$ and input mirror transmission $T_{\text {in }}$, the cavity half width at half maximum power bandwidth $\gamma / 2 \pi$, in units of $\mathrm{Hz}$, is

$$
\frac{\gamma}{2 \pi}=\frac{T_{\mathrm{in}}+\Lambda}{4 \pi} \frac{c}{2 L} \approx \frac{c}{4 L \mathcal{F}},
$$

where $\Lambda$ is the round-trip loss in the cavity and $\mathcal{F}$ is the finesse of the cavity.

From the approximation on the right, it follows that in order to achieve a bandwidth as narrow as $50 \mathrm{~Hz}$, the filter cavity must either be very long or have a very high finesse (i.e., very low optical loss). For example, a 300-m-long filter cavity like the one planned for $\mathrm{A}+[13]$ (and the one operating in Japan $[14,17]$ ) can achieve a $50 \mathrm{~Hz}$ bandwidth with a finesse of $\mathcal{F}=5000$, while a $15 \mathrm{~m}$ filter cavity would need a finesse 20 times higher, $\mathcal{F}=100000$.

Experimental setup.-An overview of the experimental apparatus is shown in Fig. 1. A 16-m-long filter cavity is enclosed in ultrahigh vacuum chambers identical to those at the LIGO sites. The input and output mirrors of the filter cavity are 2" superpolished fused-silica optics mounted on tip-tilt suspensions [18] that are placed on seismically isolated platforms that mimic those in Advanced LIGO [19]. The filter cavity storage time is $2.8 \mathrm{~ms}$ and has a finesse of $~ 80000$ for $1064 \mathrm{~nm}$ light. The inferred cavity round-trip loss, excluding input mirror transmissivity, is $\Lambda=19 \mathrm{ppm}$, corresponding to a decoherence time of $5.7 \mathrm{~ms}$ [20].

The frequency-independent squeezed vacuum source which drives the filter cavity is nearly identical to the one currently in use in Advanced LIGO [3]. Squeezing is produced by parametric down-conversion in an in-vacuum bow tie optical parametric oscillator (OPO) pumped by $532 \mathrm{~nm}$ light that is delivered via an optical fiber. The squeezed beam is then reflected off the detuned filter cavity. A Faraday isolator steers the returning squeezed beam from the filter cavity through a viewport toward an in-air balanced homodyne detector [21], where the frequencydependent squeezed state is characterized. The key parameters of this system are listed in Table I.

Observation of frequency-dependent squeezing.Figure 2 shows squeezing measurements for various configurations of the filter cavity. First, a reference measurement without squeezing is taken to determine the shot noise. All subsequent measurements are normalized to this shot-noise level. Next, a measurement of frequency-independent squeezing is taken by holding the filter cavity far from resonance (brown). The measured squeezing level of $4.4 \mathrm{~dB}$ establishes the parameters of the squeezed vacuum source, independent of the filter cavity.

All measurements of frequency-dependent squeezing shown are performed with a $\sim 30 \mathrm{~Hz}$ filter cavity detuning.
TABLE I. Experimentally determined parameters of the frequency-dependent squeezed vacuum source. Entries marked by an asterisk were determined most accurately through fitting to the data. In all cases, fitting produced values in agreement with independent measurements and their uncertainties.

\begin{tabular}{lc}
\hline \hline Parameter & Value \\
\hline Filter cavity length & $16.0611(2) \mathrm{m}$ \\
Filter cavity storage time & $2.8(1) \mathrm{ms}$ \\
Filter cavity decoherence time & $5.7(3) \mathrm{ms}$ \\
OPO nonlinear gain & $4.5(1)$ \\
OPO escape efficiency & $98(1) \%$ \\
Propagation loss* & $17(1) \%$ \\
Homodyne visibility & $91.9(4) \%$ \\
Photodiode quantum efficiency & $99(1) \%$ \\
Filter cavity round-trip loss & $19(1) \mathrm{ppm}$ \\
Frequency-independent phase noise (rms) & $10(5) \mathrm{mrad}$ \\
Filter cavity length noise (rms) & $0.7(1) \mathrm{pm}$ \\
Filter cavity mode matching & $92(1) \%$ \\
\hline \hline
\end{tabular}

With this detuning, measurements are taken at five different homodyne angles: one for measuring the squeezed quadrature at frequencies outside the filter cavity bandwidth (purple), one for measuring the antisqueezed quadrature (blue), and intermediate homodyne angles (orange, green, red).

We use a detailed quantum noise model to verify our understanding of the system and the measured parameters. In particular, we employ the two-photon formalism, introduced in Ref. [22] and expanded upon in Ref. [9], to model the action of the cavity on the squeezed state. Decoherence and degradation mechanisms arising from experimental imperfections are also included. For each measurement, parameters that are difficult to measure directly (marked with asterisks in Table I) are determined from the model fits.

Under normal operating conditions in Advanced LIGO, the filter cavity will rotate the squeezed state to compensate the optomechanical interaction in the interferometer [6]. While the current measurement does not include the LIGO interferometer, our model allows us to compute the maximum quantum noise reduction that could be measured if the interferometer's optomechanical interaction were present. This is shown in the black trace in Fig. 2. At frequencies near the rotation frequency (e.g., $30 \mathrm{~Hz}$ ), the loss in the cavity causes this model projection to go above the shot-noise level. This is expected, given the finesse and optical losses of the $16 \mathrm{~m}$ cavity [10], and is part of the motivation for the 300-m-long filter cavity for A+. Optics of similar quality in a $300 \mathrm{~m}$ filter cavity will result in little degradation of squeezing even at frequencies near the filter cavity resonance [13].

Control of frequency-dependent squeezing.-For squeezed states to be employed in gravitational-wave detectors, the phase of the squeezed states (i.e., their orientation relative to the readout quadrature) must be kept under tight control. Squeezed vacuum states present a particular challenge, as they lack a copropagating carrier 


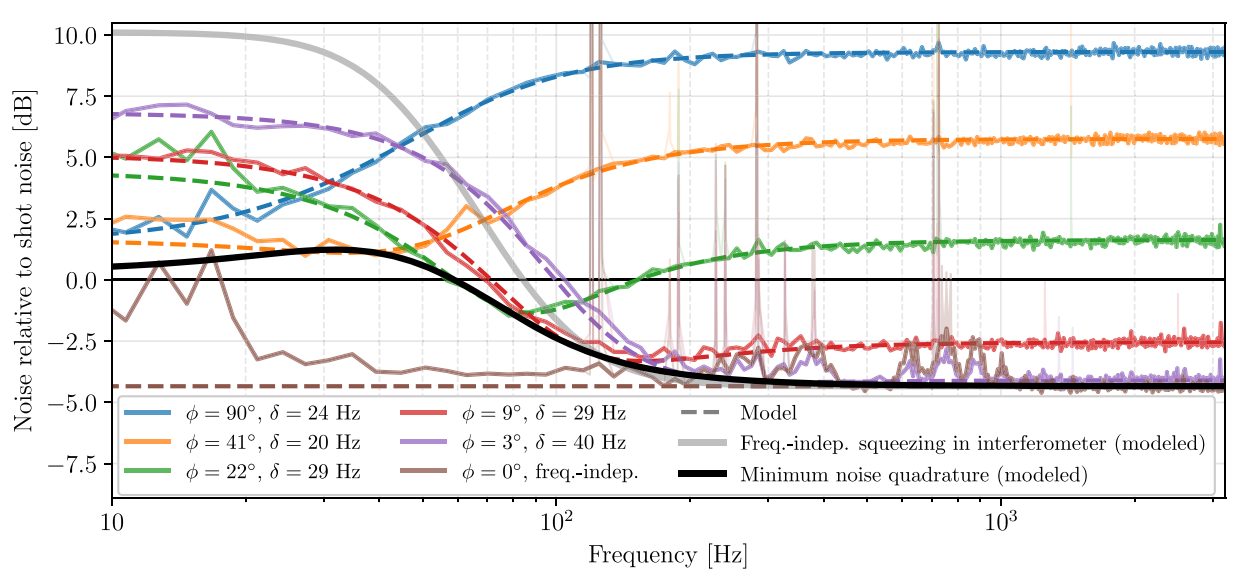

FIG. 2. Frequency-dependent squeezing at gravitational-wave detector frequencies. Measured noise (solid) is plotted alongside models (dashed). The $0 \mathrm{db}$ line refers to the shot noise level, representing unsqueezed vacuum fluctuations. Frequency-independent squeezing (brown) shows the performance of our squeezer to low frequencies without the filter-cavity (FC). The action of the cavity on the squeezed vacuum is demonstrated in both squeezed (purple) and antisqueezed (blue) quadratures, as well as intermediate homodyne angles $\phi$ (orange, green, red). The cavity detunings $\delta$ were selected to be appropriate for gravitational-wave detectors (20-40 Hz). The detuning variations are from a nonlinear relationship between the squeezing angle and operating point of the RLF control scheme. The black line shows the minimum relative quantum noise possible in an interferometer by squeezing after reflection by this cavity (detuned $30 \mathrm{~Hz}$ ). For a relative comparison, the gray curve models the quantum noise change expected from injecting only the frequencyindependent source of this experiment into a matched interferometer [6]. Data coinciding with acoustic peaks have been excluded from the frequency-binned data, but are presented in the faded traces. The turn up in each curve starting at $20 \mathrm{~Hz}$ is due to a mechanical resonance of the optical table.

field needed for phase measurements. The problem compounds with frequency-dependent squeezing, in which the cavity rotation frequency must also be controlled.

As with the frequency-independent squeezed light sources [3] currently used in Advanced LIGO, overall phase control is performed with a coherent locking field (CLF), which is injected into the OPO and copropagates with the squeezed state [23]. The CLF is offset in frequency (typically a few megahertz) relative to the carrier, in order to minimize its noise impact while allowing a highprecision measurement of the propagation phase of the squeezed state.

Deviations of the filter cavity from the nominal detuning (i.e., length offset relative to the carrier frequency) manifest in two ways. First, the rms cavity length noise causes variations in the squeezed state rotation near and below the rotation frequency, thereby allowing some of the antisqueezed quadrature to contaminate the readout. This is analogous to phase noise in frequency-independent squeezing, but degrades squeezing performance at frequencies rotated by the filter cavity. For $\mathrm{A}+$, the rms displacement noise for the filter cavity must be lower than $1 \mathrm{pm}$.

The second manifestation of filter cavity detuning noise is the modulation of any carrier field which leaks from the interferometer's readout port into the squeezed light path. This leaked "backscatter" light undermines the benefits of squeezing by adding noise to the readout in the gravitational-wave band [1,24]. Optical isolation elements are used to reduce this leakage light, but at the cost of increased optical loss that degrades squeezing. For $\mathrm{A}+$, the filter cavity length noise in the $10-100 \mathrm{~Hz}$ band must be better than $10^{-16} \mathrm{~m} / \sqrt{\mathrm{Hz}}$.

Maintaining the rotation frequency of our frequencydependent squeezing amounts to sensing and controlling the filter cavity length. Historically this has been done with a second field of a different wavelength (e.g., $532 \mathrm{~nm}$ ) which can be introduced into the squeezed path with dichroic optics [12]. We use this approach to gain initial control of the filter cavity resonance, but we find it insufficient for high-precision control, as the cavity response has subtle discrepancies between the two wavelengths. To meet the stringent noise requirements of Advanced LIGO and future detectors, we have developed a novel sensing scheme that we present here.

The filter cavity sensing scheme demonstrated in this experiment adapts the LIGO coherent control scheme [3] to prepare an additional $1064 \mathrm{~nm}$ field, frequency shifted with respect to the main interferometer carrier field so as to resonate in the filter cavity. This resonant locking field (RLF) is then sensitive to the filter cavity length, and phase or frequency noise on it will be interpreted as length noise. The RLF is generated together with the CLF via an acousticoptic modulator [see Fig. 1(a)], and both fields copropagate with the squeezed vacuum field. Copropagation is critical to avoid phase noise relative to the CLF, which is phase locked to the interferometer carrier field. We estimate the RLF scheme to suppress by at least 2 orders of magnitude the phase modulations arising from seismic and acoustic noise in alternative schemes that use a 532 or $1064 \mathrm{~nm}$ beam which do not benefit from the CLF phase control. In summary, the 
RLF scheme has two benefits. First and foremost, its phase coherence with the CLF and thus the squeezed state allow it to meet the stringent backscatter requirements for $\mathrm{A}+$. Second, it senses the cavity length at the same wavelength as the squeezing field, thereby avoiding drifts and noises found in schemes which employ other wavelengths for filter cavity control.

Conclusions and outlook.-The A+ upgrade to the Advanced LIGO detectors relies on frequency-dependent squeezing to achieve a broadband reduction of quantum noise. Achieving the requisite $<40 \mathrm{~Hz}$ rotation frequency of the squeezed state necessitates a 300-m-long filter cavity with low-loss optics. Here we produce this rotation frequency by coupling the Advanced LIGO squeezed vacuum source with a $16 \mathrm{~m}$ filter cavity, the longest available in our facility. The present result improves on a previous experiment by almost 2 orders of magnitude [25], providing assurance that the 300-m-long filter cavity currently under construction for A+ will work as intended. Just as important, we demonstrate the ability to control the filter cavity length with a novel technique capable of meeting stringent requirements imposed by backscattered light noise. When combined with improved optical mirror coatings, low-loss Faraday isolators [26], and active mode-matching elements, frequency-dependent squeezing enables a broadband improvement of a factor of 2 in the detector noise over Advanced LIGO [27], a factor of 8 in detection volume, to vastly expand LIGO's discovery space [28].

LIGO was constructed by the California Institute of Technology and Massachusetts Institute of Technology with funding from the National Science Foundation, and operates under Cooperative Agreement No. PHY-1764464. Advanced LIGO was built under Grant No. PHY-0823459. The authors gratefully acknowledge the National Science Foundation Graduate Research Fellowship under Grant No. 1122374. Several people have contributed to the experiment over the years, in particular, John Miller, Georgia Mansell, Evan Hall, Naoki Aritomi, and Vivishek Sudhir. Measurements of the optical loss of the filter cavity optics have been made by Joshua Smith, Adrian Avila-Alvarez, and Juan A. Rocha at California State University, Fullerton. We also acknowledge fruitful discussions with members of the LIGO Scientific Collaboration, Virgo, and KAGRA Collaboration.

*lee.mcculler@ligo.org

†chris.whittle@ligo.org

[1] J. Aasi et al., Classical Quantum Gravity 32, 115012 (2015).

[2] C. M. Caves, Phys. Rev. Lett. 45, 75 (1980).
[3] M. Tse, H. Yu, N. Kijbunchoo et al., Phys. Rev. Lett. 123, 231107 (2019).

[4] F. Acernese et al. (Virgo Collaboration), Phys. Rev. Lett. 123, 231108 (2019).

[5] H. Grote, K. Danzmann, K. L. Dooley, R. Schnabel, J. Slutsky, and H. Vahlbruch, Phys. Rev. Lett. 110, 181101 (2013).

[6] H. Yu et al. (LIGO Collaboration), arXiv:2002.01519.

[7] F. Acernese et al. (VIRGO Collaboration) (to be published).

[8] H. J. Kimble, Y. Levin, A. B. Matsko, K. S. Thorne, and S. P. Vyatchanin, Phys. Rev. D 65, 022002 (2001).

[9] P. Kwee, J. Miller, T. Isogai, L. Barsotti, and M. Evans, Phys. Rev. D 90, 062006 (2014).

[10] M. Evans, L. Barsotti, P. Kwee, J. Harms, and H. Miao, Phys. Rev. D 88, 022002 (2013).

[11] J. Miller, L. Barsotti, S. Vitale, P. Fritschel, M. Evans, and D. Sigg, Phys. Rev. D 91, 062005 (2015).

[12] E. Oelker, T. Isogai, J. Miller, M. Tse, L. Barsotti, N. Mavalvala, and M. Evans, Phys. Rev. Lett. 116, 041102 (2016).

[13] L. McCuller and L. Barsotti, https://dcc.ligo.org/LIGOT1800447/public.

[14] Y. Zhao et al., preceding Letter, Phys. Rev. Lett. 124, 171101 (2020).

[15] F. Y. Khalili, Phys. Rev. D 81, 122002 (2010).

[16] L. Barsotti, J. Harms, and R. Schnabel, Rep. Prog. Phys. 82, 016905 (2018).

[17] E. Capocasa, M. Barsuglia, J. Degallaix, L. Pinard, N. Straniero, R. Schnabel, K. Somiya, Y. Aso, D. Tatsumi, and R. Flaminio, Phys. Rev. D 93, 082004 (2016).

[18] B. J. J. Slagmolen, A. J. Mullavey, J. Miller, D. E. McClelland, and P. Fritschel, Rev. Sci. Instrum. 82, 125108 (2011).

[19] F. Matichard et al., Classical Quantum Gravity 32, 185003 (2015).

[20] T. Isogai, J. Miller, P. Kwee, L. Barsotti, and M. Evans, Opt. Express 21, 30114 (2013).

[21] M. S. Stefszky, C. M. Mow-Lowry, S. S. Y. Chua, D. A. Shaddock, B. C. Buchler, H. Vahlbruch, A. Khalaidovski, R. Schnabel, P. K. Lam, and D. E. McClelland, Classical Quantum Gravity 29, 145015 (2012).

[22] A. Buonanno and Y. Chen, Phys. Rev. D 64, 042006 (2001).

[23] H. Vahlbruch, S. Chelkowski, B. Hage, A. Franzen, K. Danzmann, and R. Schnabel, Phys. Rev. Lett. 97, 011101 (2006).

[24] S. S. Y. Chua et al., Classical Quantum Gravity 31, 035017 (2014).

[25] E. Oelker, G. Mansell, M. Tse, J. Miller, F. Matichard, L. Barsotti, P. Fritschel, D. E. McClelland, M. Evans, and N. Mavalvala, Optica 3, 682 (2016).

[26] E. Genin, M. Mantovani, G. Pillant, C. D. Rossi, L. Pinard, C. Michel, M. Gosselin, and J. Casanueva, Appl. Opt. 57, 9705 (2018).

[27] L. Barsotti et al., https://dcc.ligo.org/LIGO-T1800042/ public.

[28] B. P. Abbott et al., Living Rev. Relativity 21, 3 (2018). 